\title{
Strong, Ultralight Nanofoams with Extreme Recovery and Dissipation by Manipulation of Internal Adhesive Contacts
}

Sei Jin Park ${ }^{1,2}$, Jungho Shin ${ }^{3}$, Daniel J. Magagnosc ${ }^{4}{ }^{\dagger}$, Sanha Kim ${ }^{1}$, Changhong Cao ${ }^{1}$, Kevin T. Turner ${ }^{5}$, Prashant K. Purohit ${ }^{5}$, Daniel S. Gianola ${ }^{3}$, and Anastasios John Hart ${ }^{1, *}$

${ }^{1}$ Department of Mechanical Engineering, Massachusetts Institute of Technology, Cambridge, Massachusetts 02139, USA

${ }^{2}$ Physical and Life Sciences Directorate, Lawrence Livermore National Laboratory, 7000 East Ave, Livermore, California, 94550, USA

${ }^{3}$ Materials Department, University of California Santa Barbara, Santa Barbara, California, 93106, USA

${ }^{4}$ Materials Science and Engineering, University of Pennsylvania, Philadelphia, Pennsylvania 19104, USA

${ }^{5}$ Department of Mechanical Engineering and Applied Mechanics, University of Pennsylvania, Philadelphia, Pennsylvania 19104, USA

†Current address: U.S. Army Research Laboratory, Aberdeen Proving Ground, Maryland, 21005, USA

*Email: ajhart@mit.edu

\section{Experimental setup}

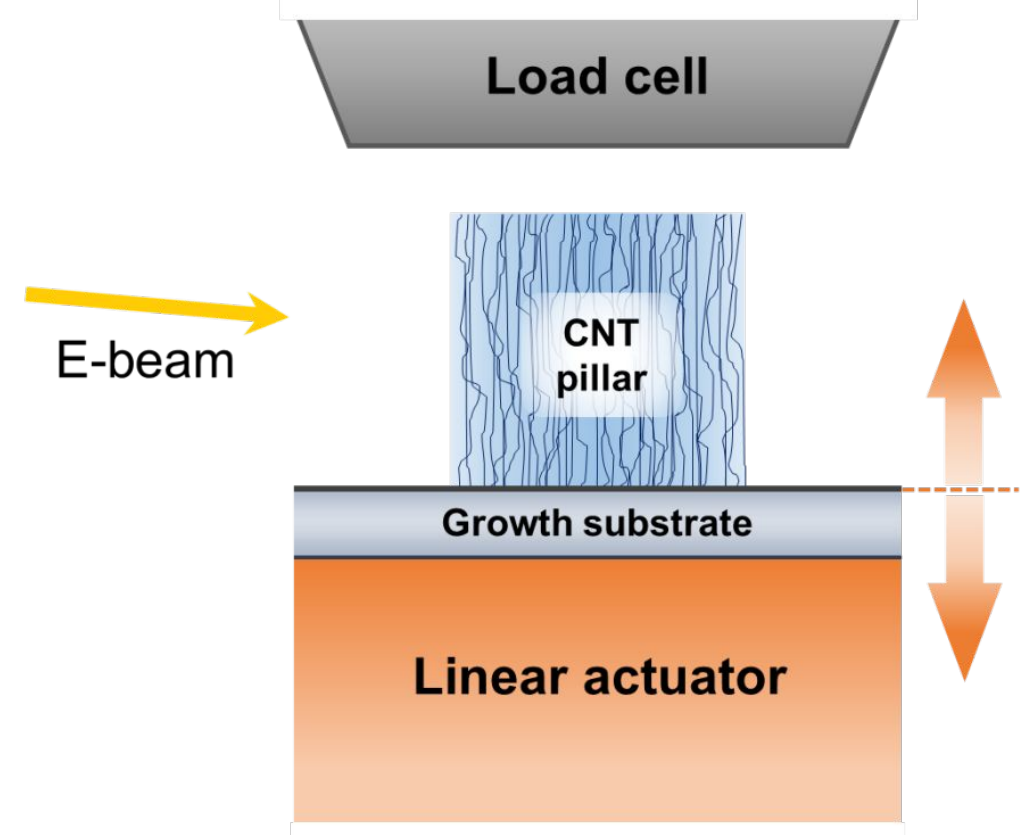

Figure S1. A schematic representation of the uniaxial compression experiments performed on the CNT 
pillars and ceramic-CNT foams.

\section{Alumina layer thickness measurements by HRTEM}

a

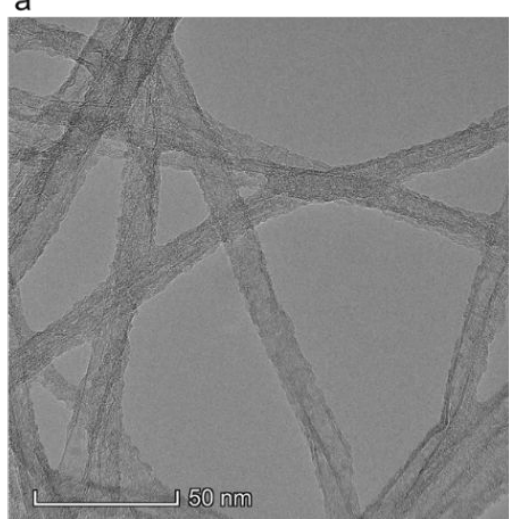

b

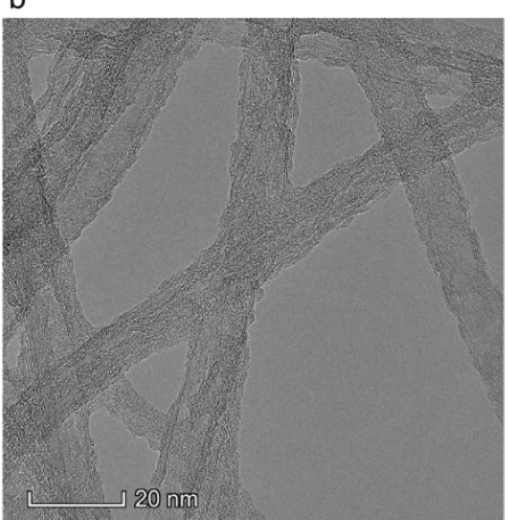

C

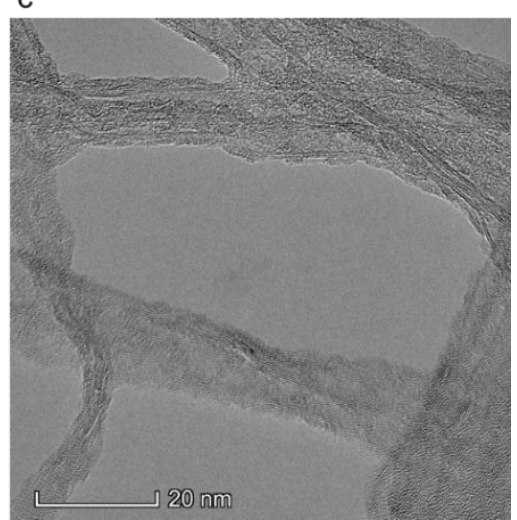

Figure S2. TEM micrographs of alumina (ALD 2 cycles) coated CNTs. The coating layer does not fully cover the CNTs yet, and the coated area has high roughness.
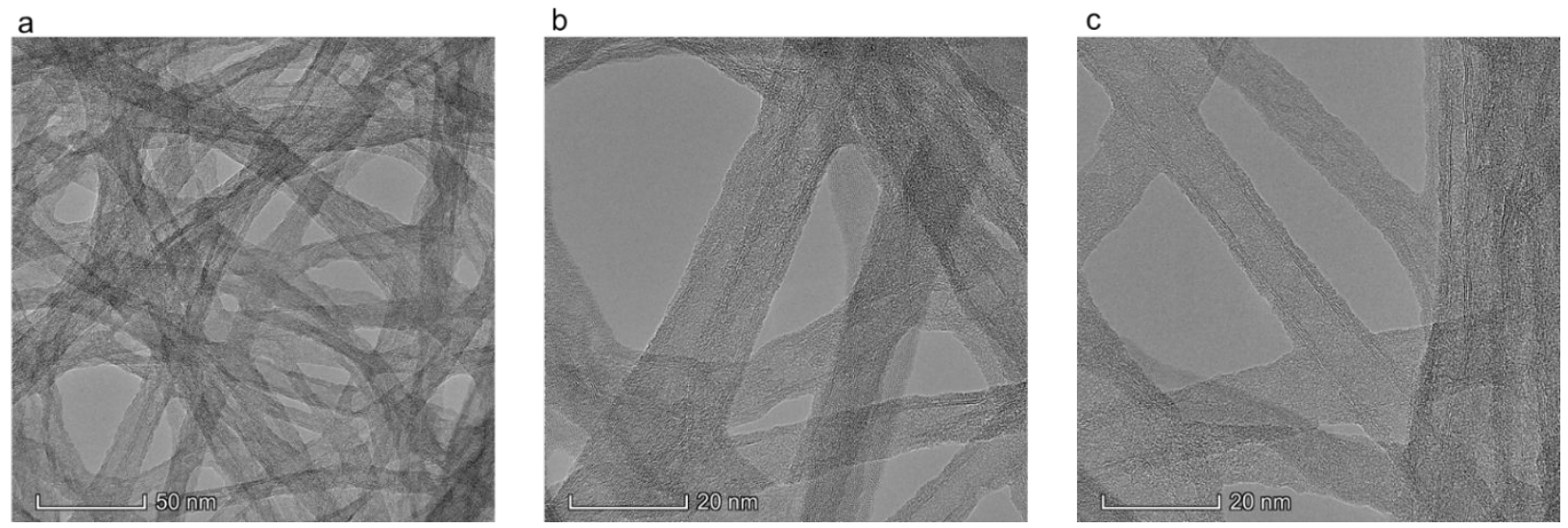

Figure S3. TEM micrographs of alumina (ALD 5 cycles) coated CNTs. The alumina layer fully covers the CNTs but are not very uniform yet.

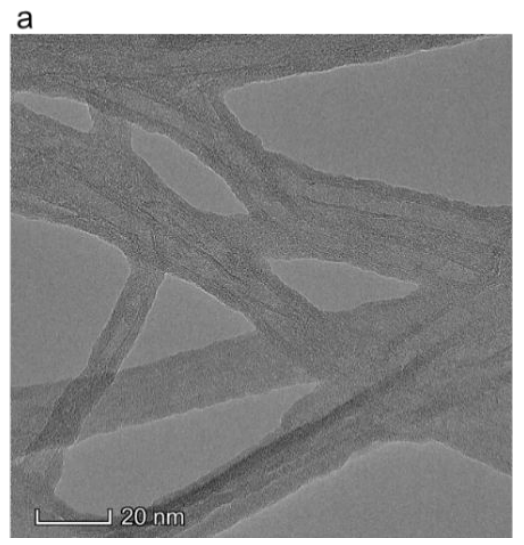

b

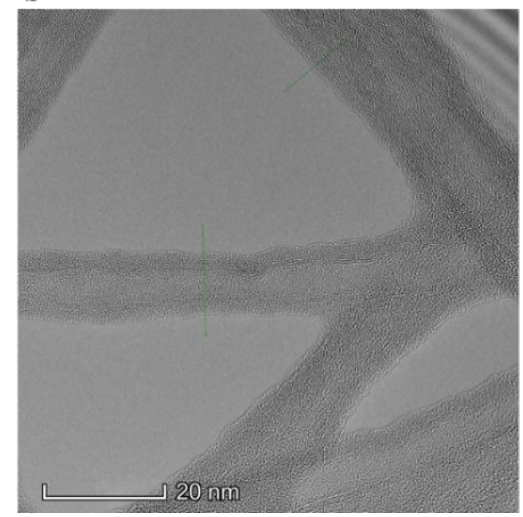

C

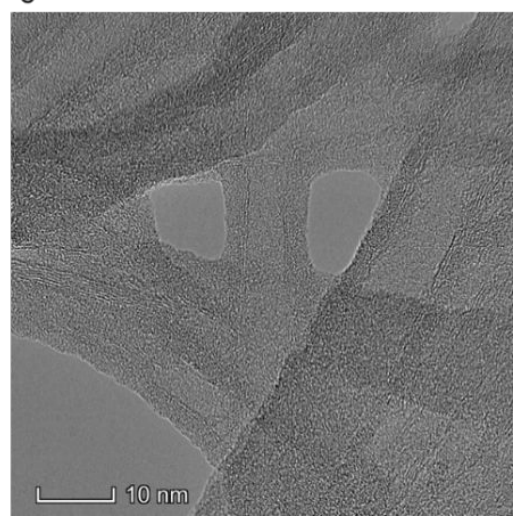


Figure S4. TEM micrographs of alumina (ALD 10 cycles) coated CNTs. The alumina layer is more uniform.
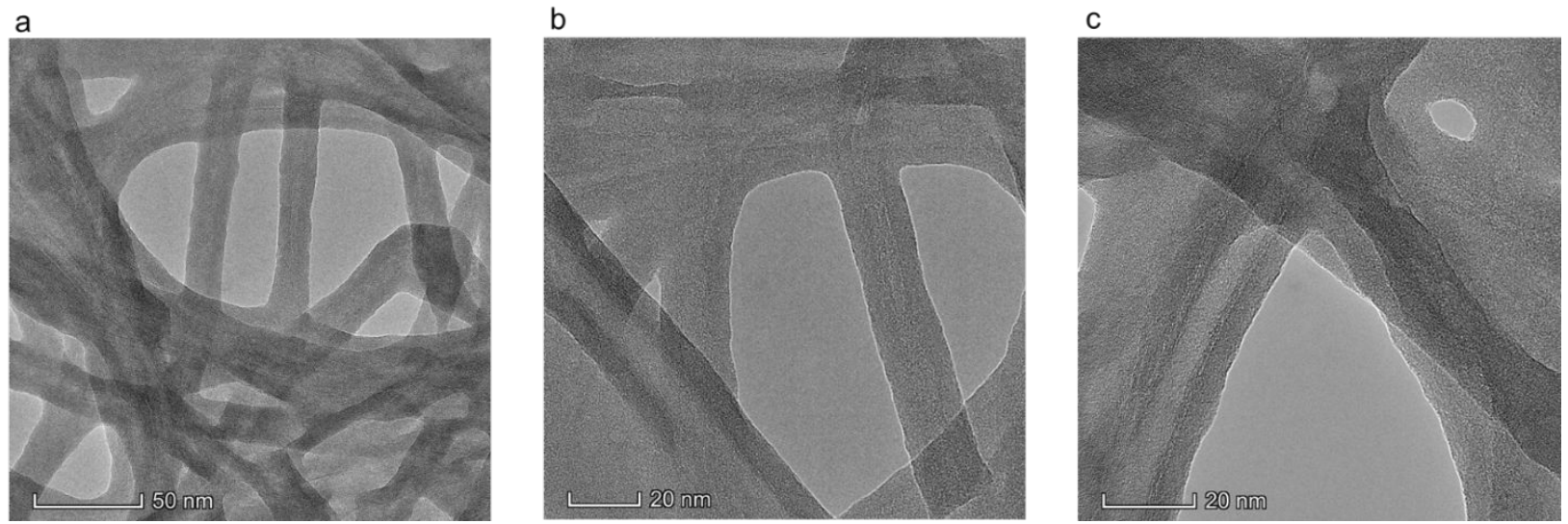

Figure S5. TEM micrographs of alumina (ALD 20 cycles) coated CNTs. CNTs become harder to observe as the coating becomes thicker.

Table S1. ALD alumina layer thickness measured from TEM micrographs

\begin{tabular}{llll}
\hline & $\mathbf{5}$ cycles & $\mathbf{1 0}$ cycles & $\mathbf{2 0}$ cycles \\
\hline $\mathbf{N}$ & 34 & 38 & 30 \\
Mean & 1.09 & 2.12 & 5.30 \\
STDEV & 0.15 & 0.34 & 0.27 \\
Min. & 0.71 & 1.60 & 4.53 \\
Max. & 1.37 & 2.99 & 5.81 \\
\hline
\end{tabular}




\section{Internal adhesion caused by e-beam exposure}

When compression was performed with the e-beam on at high magnification, the unloaded ceramic-CNT foam appears to tilt forward toward the viewer (Figure S6,7). This tilt is absent when compression experiments were performed while minimizing the pillar's exposure to the electron beam by lowering the magnification. We found that, for all coating conditions, the recovery was enhanced by the absence of exposure to the electron beam. A possible explanation is that the alumina layer stores remnant charge on the surfaces exposed to incident electrons, and these negatively charged struts adhere to the neighboring uncharged struts when they come into contact upon compression via electrostatic interactions. This interpretation is consistent with the observation that the CNT pillars bend forward after unloading, owing to the electron beam-irradiated surface recovering less than the rest of the structure that did not experience the same electron dose. While this observation further suggests that self-adhesive interactions (here, mediated by charge) influence the recovery after compression, all subsequent testing was done without exposure to the electron beam.

\section{Ceramic-CNT foam ( $\mathrm{t}_{\mathrm{AIOx}}=1.1 \mathrm{~nm}$, normal e-beam exposure)}
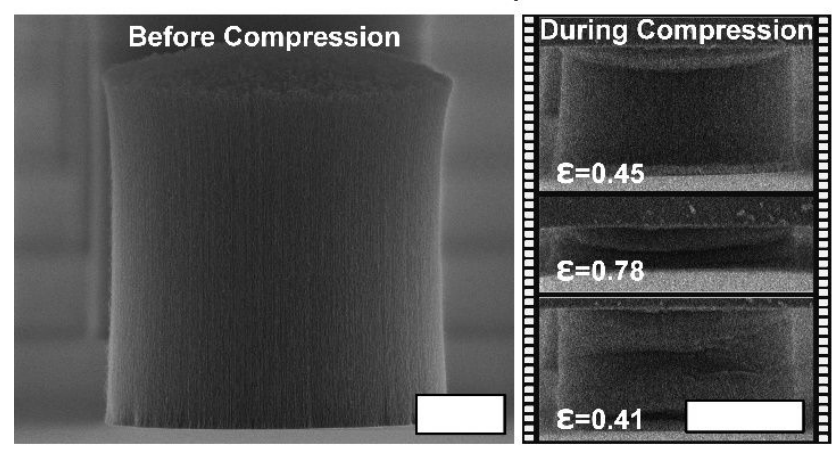

After Compression

Figure S6. Compression of ceramic-CNT foam with normal e-beam exposure exhibiting high recovery with a few creases evident, scale bars $=5 \mu \mathrm{m}$ (before), $10 \mu \mathrm{m}$ (during), and $5 \mu \mathrm{m}$ (after).

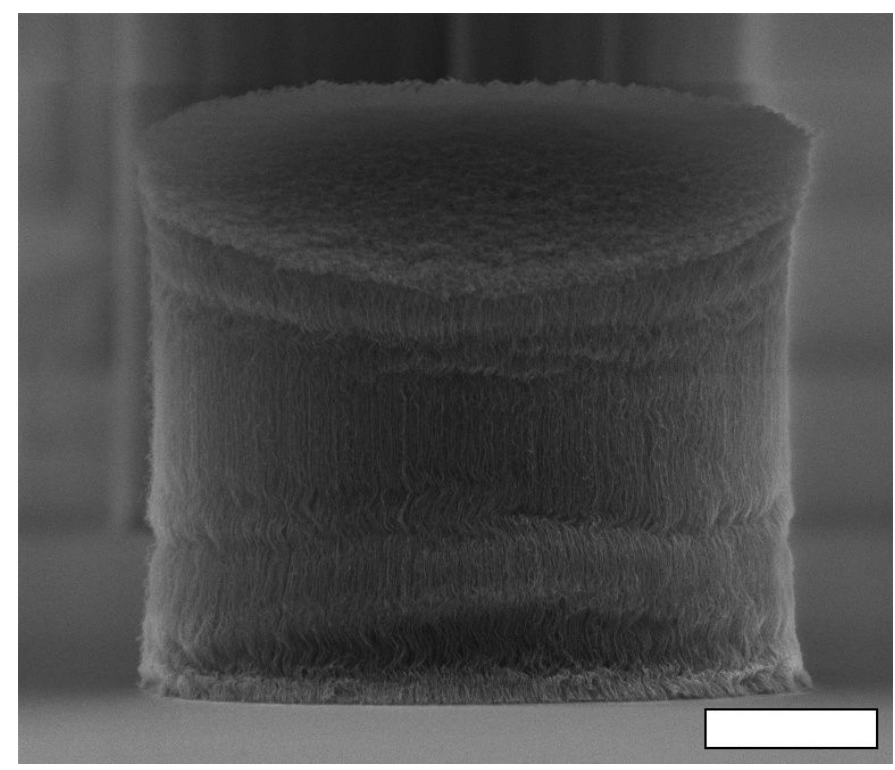


Figure S7. Ceramic-CNT foam $\left(t_{A l O x}=1.1 \mathrm{~nm}\right)$ tilts forward after compression, scale bar $=5 \mu \mathrm{m}$.

\section{Atomic force microscopy (AFM) pull off force measurements}

AFM deflection tests were performed on CNT pillars and ceramic-CNT foams $\left(t_{A l O x}=2.1 \mathrm{~nm}\right)$ using Park Systems model XE7. A silicon dioxide colloidal AFM probe (NanoAndMore Inc.) with a sphere size of 15 $\mu \mathrm{m}$ and a spring constant of $32 \mathrm{~N} / \mathrm{m}$ was used for deflection tests at a constant loading/unloading rate of $0.5 \mu \mathrm{m} / \mathrm{s}$. At each normal load, 10 deflection tests were performed at random locations in a $100 \mu \mathrm{m}^{2}$ area on the forest top surface (Figure S8a) and the pull-off force was measured from each unloading curve as shown in Figure S8b. The overlap of the loading and unloading curves of the ceramic-CNT foams indicates a recoverable deformation ${ }^{1}$ (Figure S8c) while residual deformation was present for CNT pillars (Figure S8d). After 10 deflection tests at each normal load, the AFM tip was moved to a different region on the sample far away from the previous site to avoid repeated loading of the same location. The pull-off force increases as the normal load increases for CNT pillars but remains nearly constant for the ceramic-CNT foams as the normal force varies from $100 \mathrm{nN}$ to $3000 \mathrm{nN}$ (Figure S9).
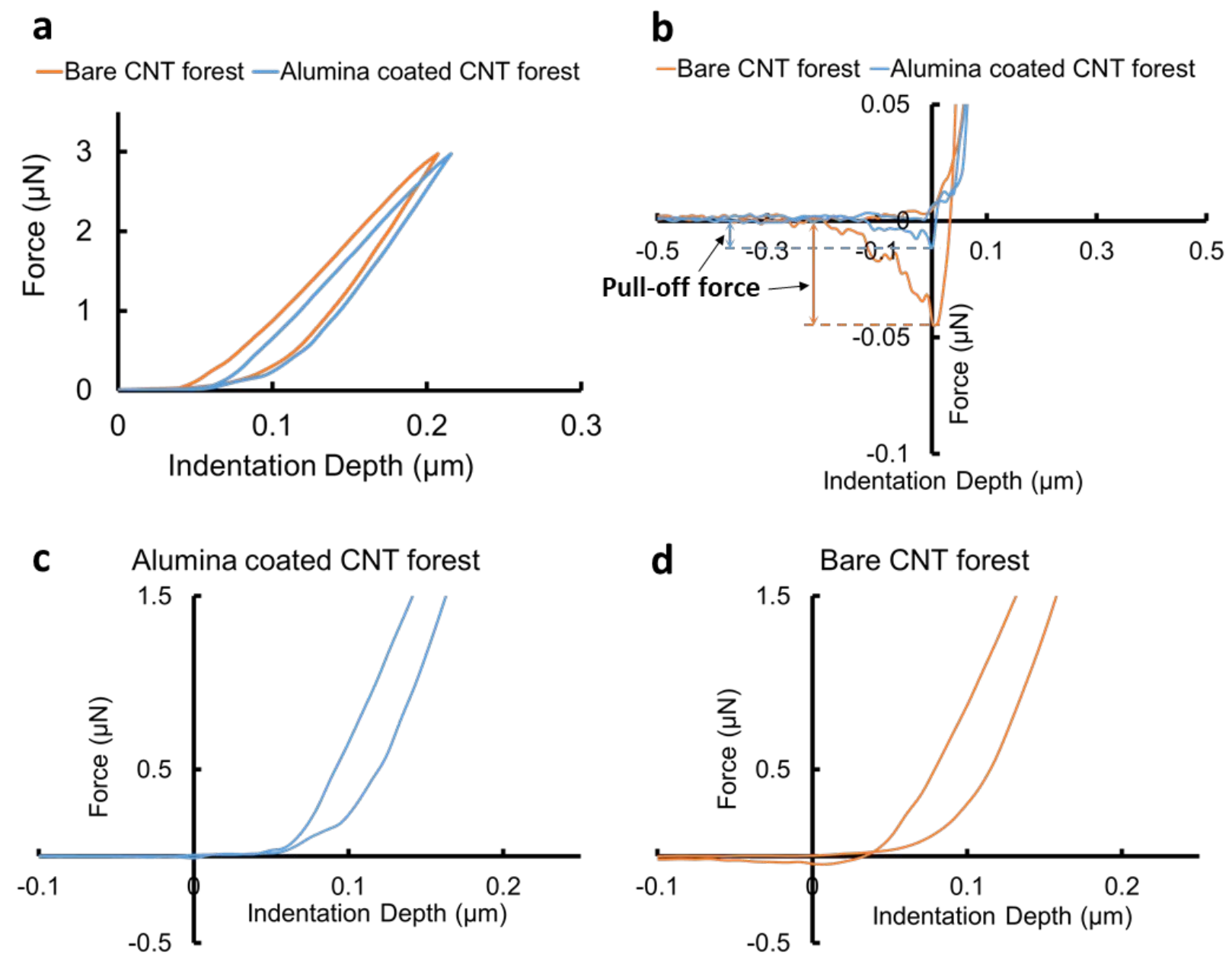

Figure S8. Representative force versus indentation depth curves for CNT pillars and ceramic-CNT foams. a) overall shape of the curves; b) close up showing how pull-off forces were measured; unloading curve 
overlaps loading curve for c) ceramic-CNT foams, but not for d) CNT pillars.

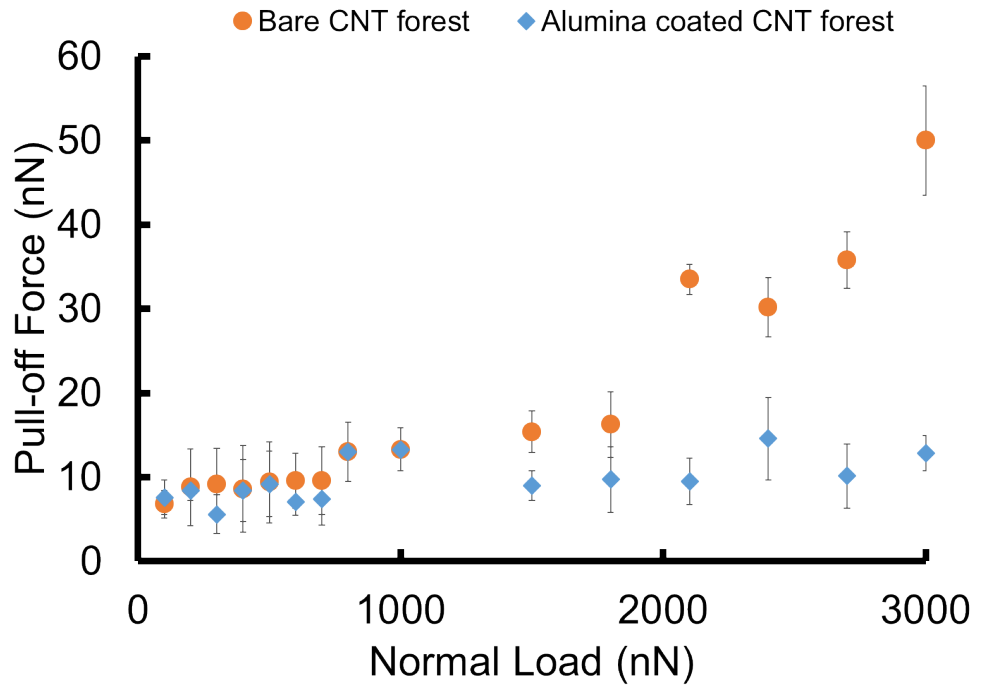

Figure S9. Pull off force measured by AFM from top surfaces of CNT pillars and ceramic-CNT foams. 


\section{Strain-rate dependence of recovery}

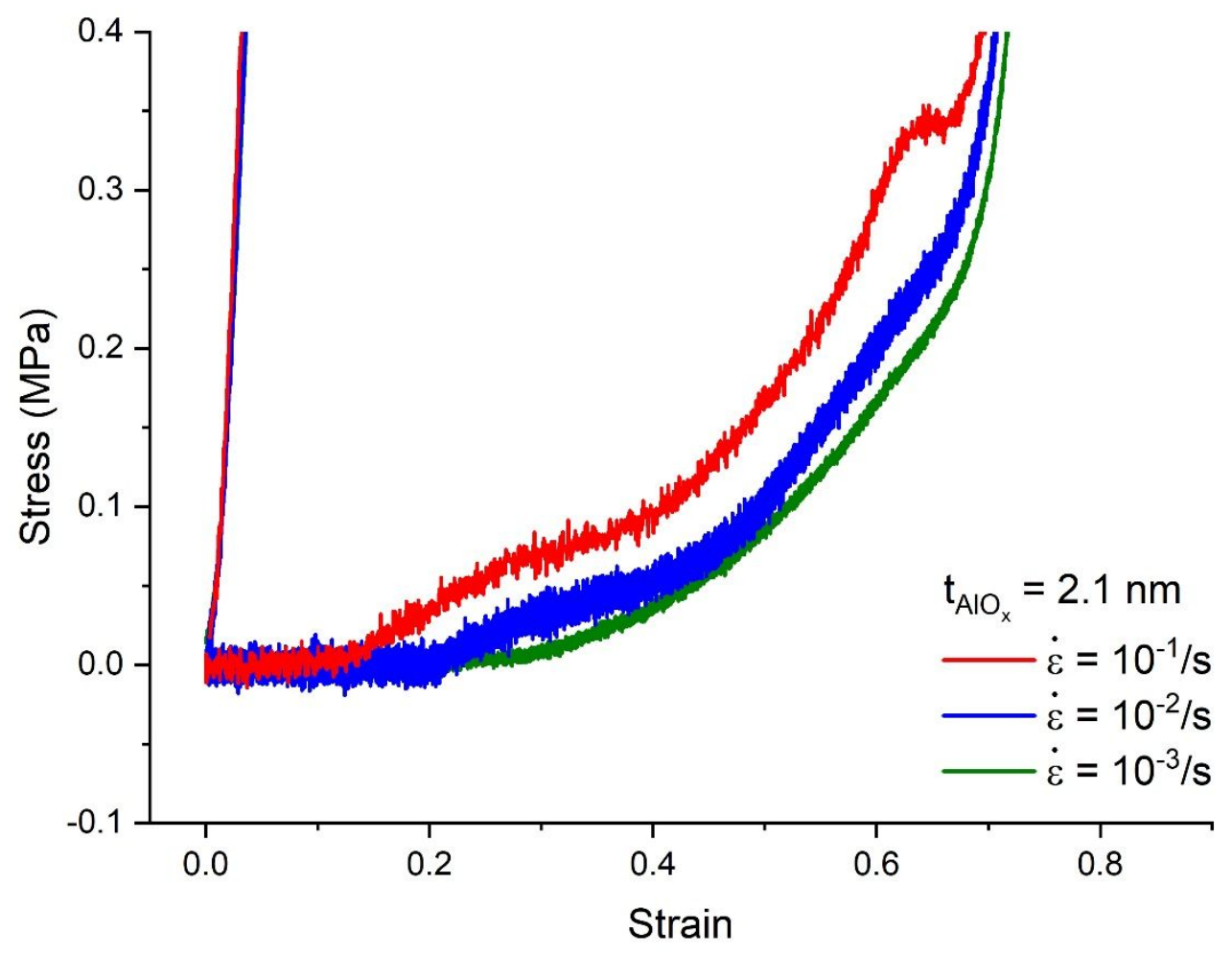

Figure S10. Unloading curves of ceramic-CNT foams $\left(t_{A l O x}=2.1 \mathrm{~nm}\right)$ at $10^{-1} / \mathrm{s}, 10^{-2} / \mathrm{s}$ and $10^{-3} / \mathrm{s}$ strain rates. The strain at which the load drops to zero is smaller as the strain rate increases. 


\section{CNT unit cell description}

Based on analysis of the approximate CNT alignment and density by small-angle X-ray scattering, we consider a unit cell height of $167 \mathrm{~nm}$ and width of $100 \mathrm{~nm} .^{2}$ At $80 \%$ compression, while preserving local alignment of the CNTs due to coordinated buckling, ${ }^{3}$ the unit cell width reduces to $20 \mathrm{~nm}$. The strut deflection is assumed to be $1 \mathrm{~nm}$ before contact, considering that the CNTs are on average $10 \mathrm{~nm}$ in diameter and the addition of alumina coating will further decrease the surface to surface distance to less than $5 \mathrm{~nm}$, and that the CNTs are not perfectly parallel. The distance between the cylinder surfaces upon contact is assumed to be $0.335 \mathrm{~nm}$ corresponding to the spacing between individual layers in graphite, and for parallel contact, the contact length is assumed to be $10 \mathrm{~nm}$. 


\section{$\underline{\text { Relationship between mobility parameters and residual strain after unloading }}$}

Here we appeal to the specialized form of the Abeyaratne-Knowles theory ${ }^{4}$ for phase transforming material in which the densified phase also behaves linearly with the same Young's modulus as the rarefied phase. We make this simplification for the sake of analytical tractability. Hence the stress-strain laws for the rarefied and densified phases are respectively,

$$
\Gamma_{L}(\sigma)=\frac{\sigma}{E^{\prime}}, \Gamma_{H}(\sigma)=\frac{\sigma}{E}+\gamma_{T}
$$

where $\mathrm{E}$ is the Young's modulus of both the rarefied and densified phases and $\gamma_{T}$ is a constant transformation strain which is on the order of 0.7 for our CNT foams. The expression for the driving force in this case is

$$
\mathrm{f}=\left(\sigma-\sigma_{0}\right) \gamma_{T}
$$

where $\sigma_{0}$ is the Maxwell stress and the kinetic law during loading and unloading takes a simple form. In particular, for unloading

$$
\bar{\Phi}=M_{H L} \gamma_{T}\left(\sigma-\sigma_{r}^{H L}\right)
$$

where $\sigma_{r}^{H L}$ is the stress at which a nucleated phase boundary starts to move. In most of our calculations with the non-linear stress-strain law for the densified phase we have taken

$$
f_{r}^{H L}=1.02 f^{H L}
$$

where $f^{H L}$ is the driving force at which the rarefied phase is nucleated in the densified phase. This ensures that the phase boundary moves as soon as it is nucleated. Note that $\sigma_{r}^{H L}=1.02 \sigma^{H L}-0.2 \sigma_{0}$. With the above simple form of the stress-strain relation and kinetic law, Abeyaratne and Knowles give an analytical solution to the governing equation ${ }^{5}$ for the stress as a function of time:

$$
\sigma=\sigma_{r}^{H L}-E v+\left(\sigma^{H L}-\sigma_{r}^{H L}+E v\right) e^{\frac{\delta-\delta^{H L}}{v L}}
$$

where $v=\frac{\dot{\delta}}{E M_{H L} \gamma_{T}^{2}}$ is a dimensionless elongation rate and $\mathrm{L}$ is the height of our sample. We are particularly interested in the residual strain, $\frac{\delta_{\text {res }}}{L}$ at $\sigma=0$ during unloading. This can be solved by setting $\sigma=0$ as

$$
\varepsilon_{r e s}=\frac{\delta_{r e s}}{L}=\varepsilon^{H L}+v \log \frac{E v-\sigma_{r}^{H L}}{E v-\sigma_{r}^{H L}+\sigma^{H L}}
$$

In our experiments the $E v$ term is negligible in comparison to $\sigma_{r}^{H L}$, so we can neglect it in the numerator on the right-hand side. Hence,

$$
\varepsilon_{r e s}=\varepsilon^{H L}+\frac{\dot{\delta}}{E M_{H L} \gamma_{T}^{2}} \log \frac{\sigma_{r}^{H L}}{\sigma_{r}^{H L}-\sigma^{H L}}
$$


a

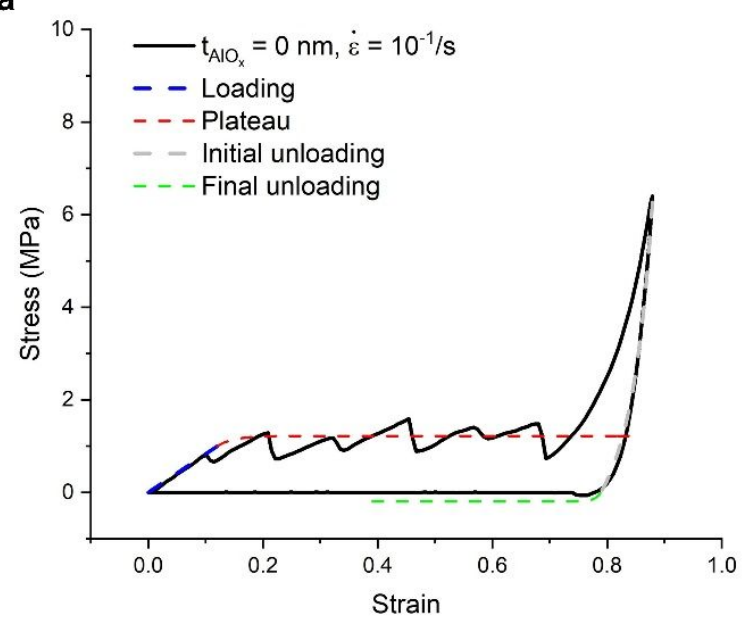

b

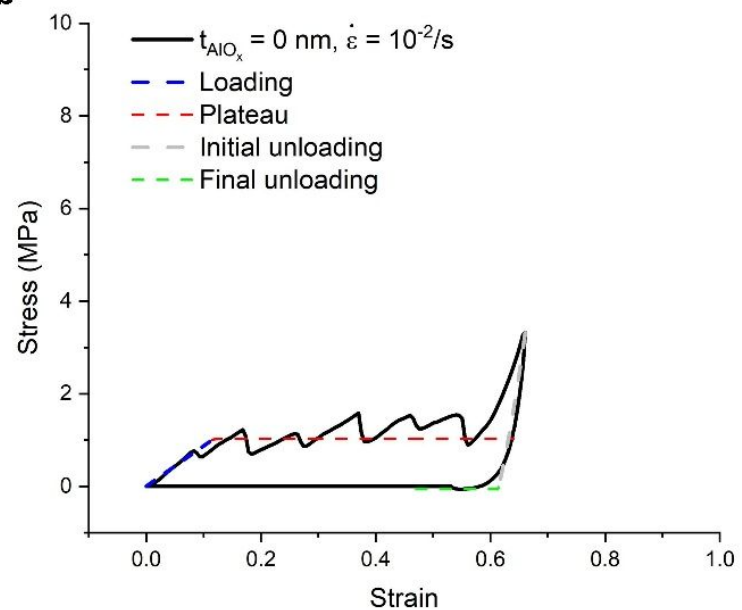

C

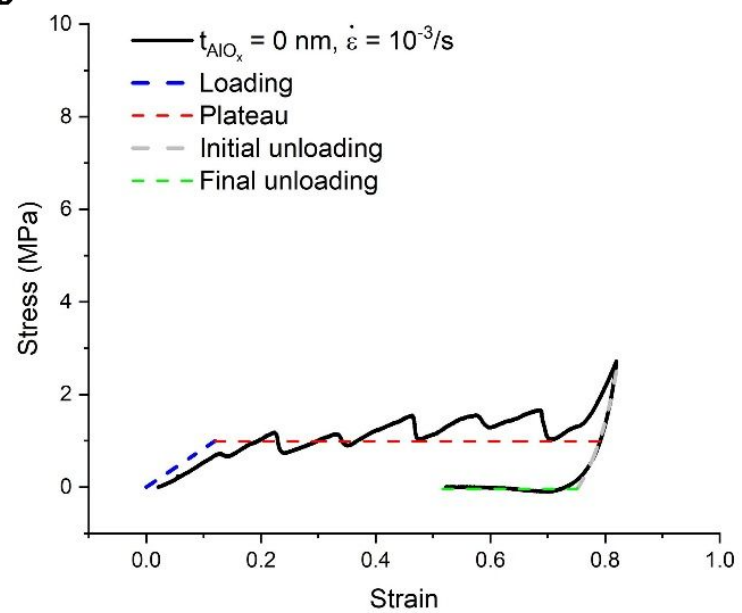

d

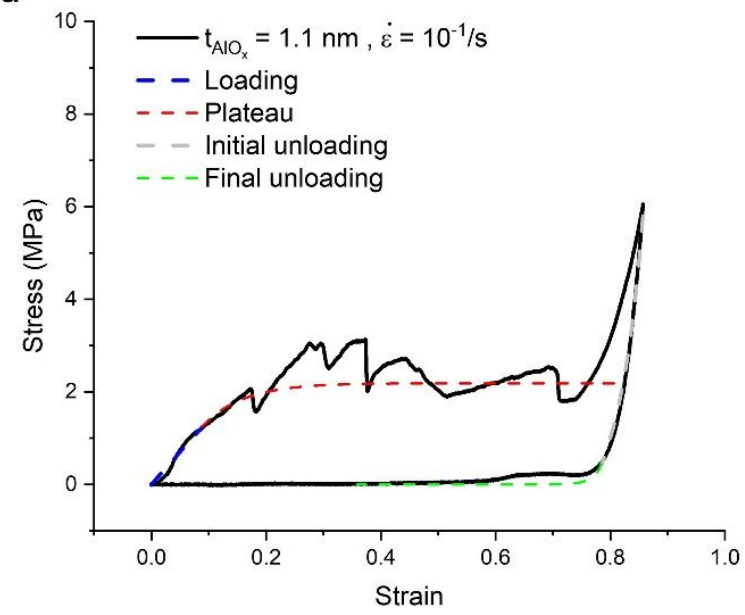

e

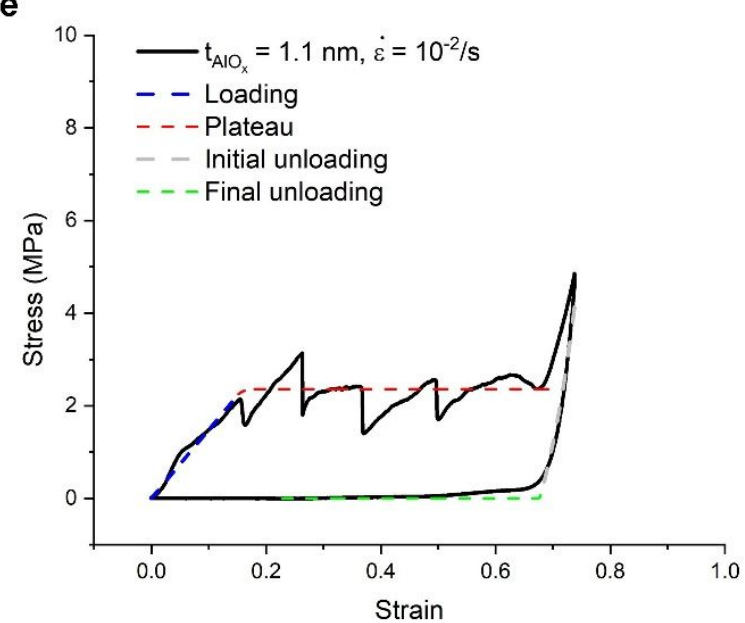

f

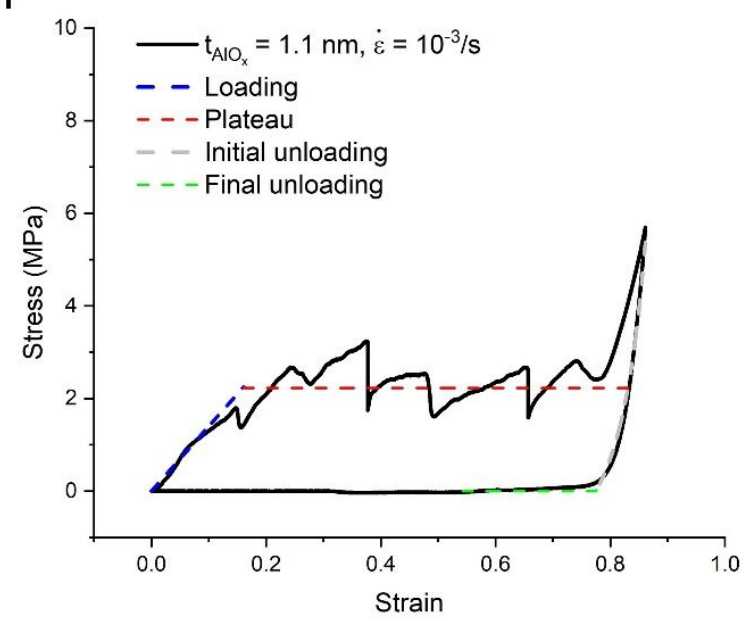

Figure S11. Compression data fitting using the phase transition model for CNT pillars with strain rates: a) $\left.10^{-1} / \mathrm{s}, \mathrm{b}\right) 10^{-2} / \mathrm{s}$, and c) $10^{-3} / \mathrm{s}$, and ceramic-CNT foams $\left(t_{A l O x}=1.1 \mathrm{~nm}\right)$ with strain rates: d) $10^{-1} / \mathrm{s}$, e) $10^{-}$ $2 / \mathrm{s}$ and f) $10^{-3} / \mathrm{s}$. 
a

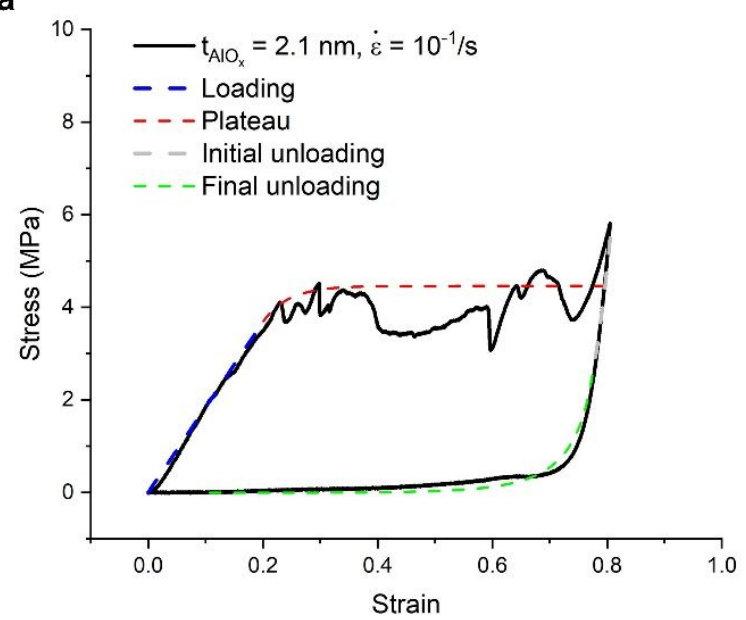

b

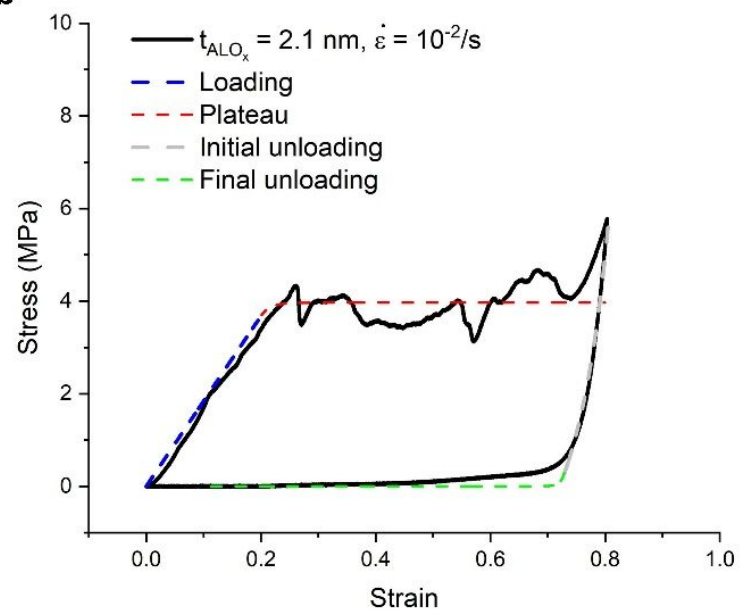

C

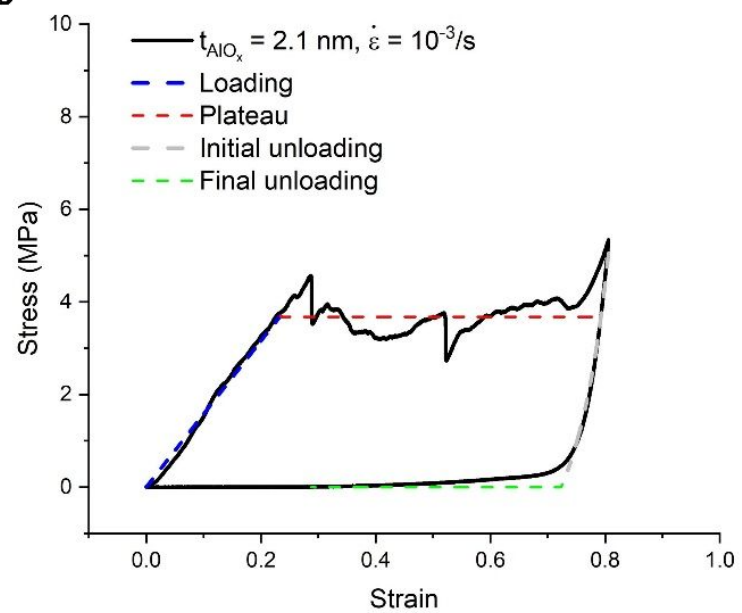

d

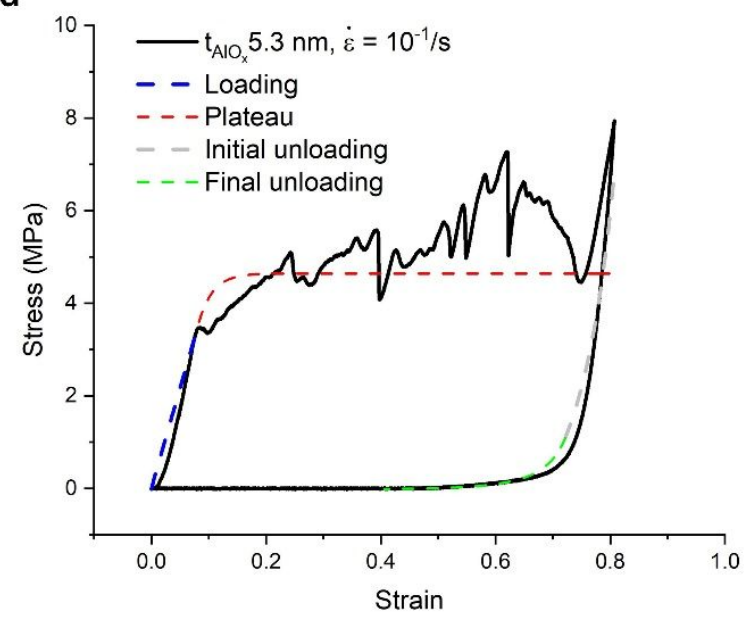

e

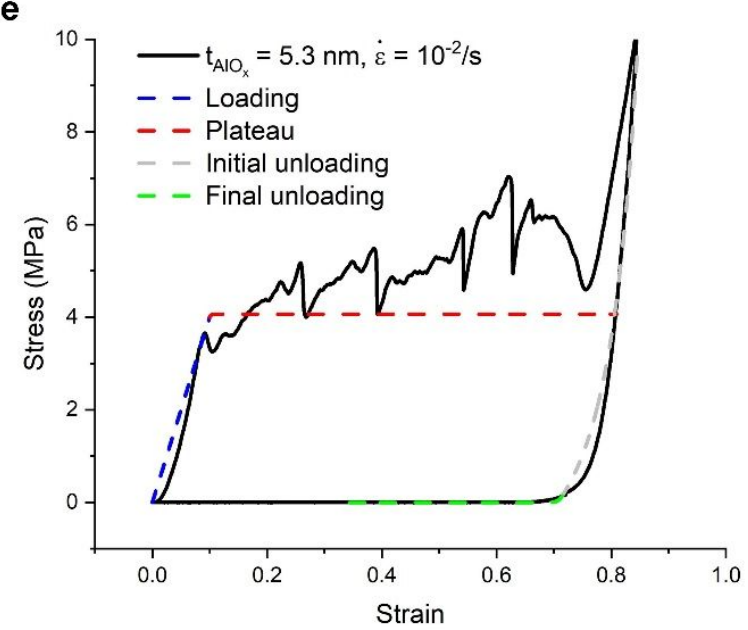

f

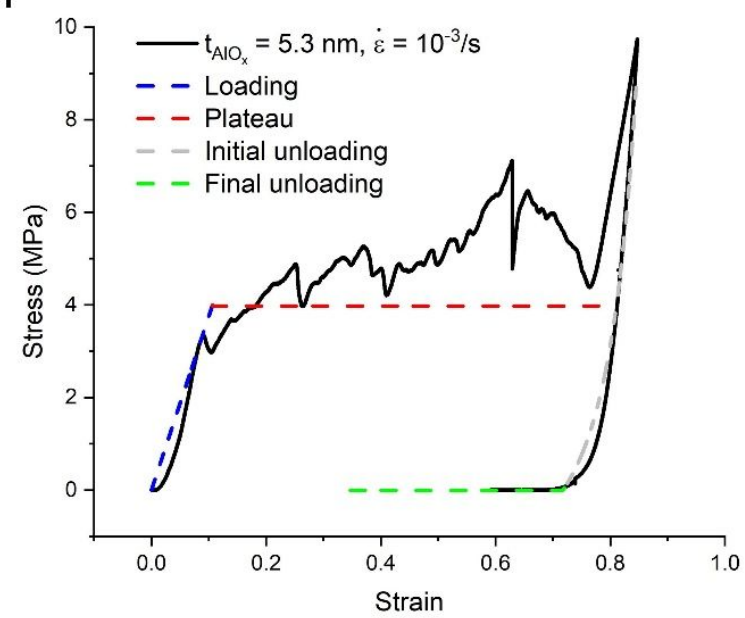

Figure S12. Compression data fitting using the phase transition model for ceramic-CNT foams $\left(\mathrm{t}_{\mathrm{AlOx}}=2.1\right.$ $\mathrm{nm}$ ) with strain rates: a) $10^{-1} / \mathrm{s}$, b) $10^{-2} / \mathrm{s}$, and c) $10^{-3} / \mathrm{s}$, and ceramic-CNT foams $\left(t_{A l O x}=5.3 \mathrm{~nm}\right)$ with strain rates: d) $10^{-1 /} / \mathrm{s}$, e) $10^{-2} / \mathrm{s}$ and f) $10^{-3} / \mathrm{s}$. 


\section{$\underline{\text { References }}$}

1. Wei, X. D.; Meng, Z. X.; Ruiz, L.; Xia, W. J.; Lee, C.; Kysar, J. W.; Hone, J. C.; Keten, S.; Espinosa, H. D., Recoverable Slippage Mechanism in Multilayer Graphene Leads to Repeatable Energy Dissipation. Acs Nano 2016, 10, 1820-1828.

2. Brieland-Shoultz, A.; Tawfick, S.; Park, S. J.; Bedewy, M.; Maschmann, M. R.; Baur, J. W.; Hart, A. J., Scaling the Stiffness, Strength, and Toughness of Ceramic-Coated Nanotube Foams into the Structural Regime. Adv. Funct. Mater. 2014, 24, 5728-5735.

3. Pathak, S.; Mohan, N.; Decolvenaere, E.; Needleman, A.; Bedewy, M.; Hart, A. J.; Greer, J. R., Local Relative Density Modulates Failure and Strength in Vertically Aligned Carbon Nanotubes. Acs Nano 2013, 7 , 8593-8604.

4. Abeyaratne, R.; Knowles, J. K., Evolution of Phase Transitions: A Continuum Theory; Cambridge University Press: Cambrige, England, 2006.

5. $\quad$ Liang, X. J.; Shin, J. H.; Magagnosc, D.; Jiang, Y. J.; Park, S. J.; Hart, A. J.; Turner, K.; Gianola, D. S.; Purohit, P. K., Compression and Recovery of Carbon Nanotube Forests Described as a Phase Transition. Int. J. Solids Struct. 2017, 122, 196-209. 